Kurt Brauchli • Hermann Oberli • Nina Hurwitz •

Klaus-Dieter Kunze • Gunter Haroske • Gernot Jundt •

Gerhard Stauch • Lech Banach • Mark Wirdnam •

Michael Mihatsch $\cdot$ Martin Oberholzer

\title{
Diagnostic telepathology: long-term experience of a single institution
}

Received: 28 October 2003 / Accepted: 15 January 2004 / Published online: 12 March 2004

(C) Springer-Verlag 2004

\begin{abstract}
Objectives: The paper reviews the development of the application of telepathology in a department of surgical pathology between 1991 and 2003. The goal of the efforts during this time was to give up the concept of programming a single application, available only between two fixed workstations with sophisticated devices and special software, and to find the virtual "largest common denominator" for implementing as many different applications as possible with the same basic system. Methods: A new telepathology system was designed as a clientserver system with a relational database at its centre. The clients interact together by transferring the questions (texts and images) to a record (case) in the database on the server and by transferring the answers to the same record on the database. Results: The new "open" telepathology system iPath (http://telepath.patho.unibas.ch) has been very well accepted by many groups around the world. The main application fields are: consultations between pa-
\end{abstract}

K. Brauchli $\cdot$ N. Hurwitz $\cdot$ G. Jundt $\cdot$ M. Wirdnam $\cdot$ M. Mihatsch · M. Oberholzer (

Department of Pathology of the University, Basel, Switzerland

e-mail: moberholzer@uhbs.ch

Tel.: +41-61-2652525

Fax: +41-61-2653194

H. Oberli

National Referral Hospital,

Honiara, Solomon Islands

K.-D. Kunze

Institute of Pathology, Technical University,

Dresden, Germany

G. Haroske

Institute of Pathology,

Kreiskrankenhaus Dresden-Friedrichsstadt,

Dresden, Germany

G. Stauch

Institute of Pathology,

Aurich, Germany

L. Banach

Institute of Pathology of the University of Transkei,

Umtata, South Africa thologists and medical institutions without a pathologist (e.g. for frozen section diagnoses or for surgical diagnoses in hospitals in South Asia or Africa), tumour boards, field studies and distance education (http://teleteach.patho. unibas.ch). Conclusions: Having observed that with iPath we have succeeded in satisfying all our telepathology needs, we are inclined to put the emphasis on the nature of the tasks being performed, as opposed to the methods or technical means for performing a given task. The three organisation models proposed by Weinstein et al. (2001) [24] can be reduced to only two models: the model of discussion groups and the model of expert groups (virtual institutes).

Keywords Telepathology · Surgical pathology ·

Telemedicine

\section{Development of telepathology in a department of surgical pathology}

The first application of telepathology at the Department of Pathology of the University of Basel in Switzerland was in 1991 [17], implementing a remote diagnosis service on intraoperative frozen sections for the Regional Hospital in Samedan, Oberengadin, Switzerland.

The telepathology system used today (iPath) is an Internet-based system (http://telepath.patho.unibas.ch) and was developed at the Department of Pathology of the University of Basel on the background of over 10 years experience. Current applications include pathology telediagnosis for hospitals, primarily in Asia, and for second-opinion consultations in subspecialities (histopathology, radiology), primarily in Europe. Our experiences have demonstrated that a multi-purpose telemedical system can also comfortably be used for tumour boards, health networks, field studies and even distance learning (http://teleteach.patho.unibas.ch).

The current challenges in telepathology are: (1) the creation of minimal standards allowing the interaction between different systems $[4,11,25]$ and (2) the organi- 
sation of individual workflow to allow efficient use of telepathology in daily routine (Brauchli et al. unpublished data). Therefore, we would like to achieve the following: (1) to answer the question, "Which are the essential fundaments of a modern, user-friendly telepathology in the future?" and (2) to summarise our observations and own experiences in telepathology and telemedicine.

\section{Essential fundaments for a modern telepathology (telemedicine)}

Telepathology is a structured dialogue

Modern computer and network technology offers all the elements for telecollaboration in medicine. For that reason, the development of telemedical systems is currently more focused on how to organise cooperation and how to use the existing technology in a most user-friendly way.

The goal of any kind of telemedicine is to exchange information. Such an exchange of information has to be possible from anywhere at any time, without delay and without additional effort. Basically, any exchange of information in medicine can be considered as a question, part of which often consists of pictures and the answer to that question. We use the terms "expert" and "non-expert" to define the roles of the partners within a real telemedical exchange of information, thus referring to who is asking the question ("non-expert") and who is delivering the answer ("expert").

Free exchange of information in medicine can be guaranteed by a client-server system

A server (Fig. 1: platform) contains a database connected with a content management system and manages the necessary connections between the clients (experts and non-experts) (Fig. 1). The server is placed within the Internet when connectivity beyond the local network is needed (Fig. 2). The members of the different discussion groups (experts and non-experts) working on the cases (by asking and answering) are all clients. A client application can be a remote microscopy session (synchronous applications) $[1,22]$ or a standard web-browser or email client for case discussion (asynchronous applications) $[1,22]$.

A modern telepathology system should have a modular structure

The experiences in the field of telepathology have demonstrated how important it can be to quickly create a new telepathological (telemedical) connection or to adapt an existing system to new user needs or new technologies. This goal can be achieved through modu-

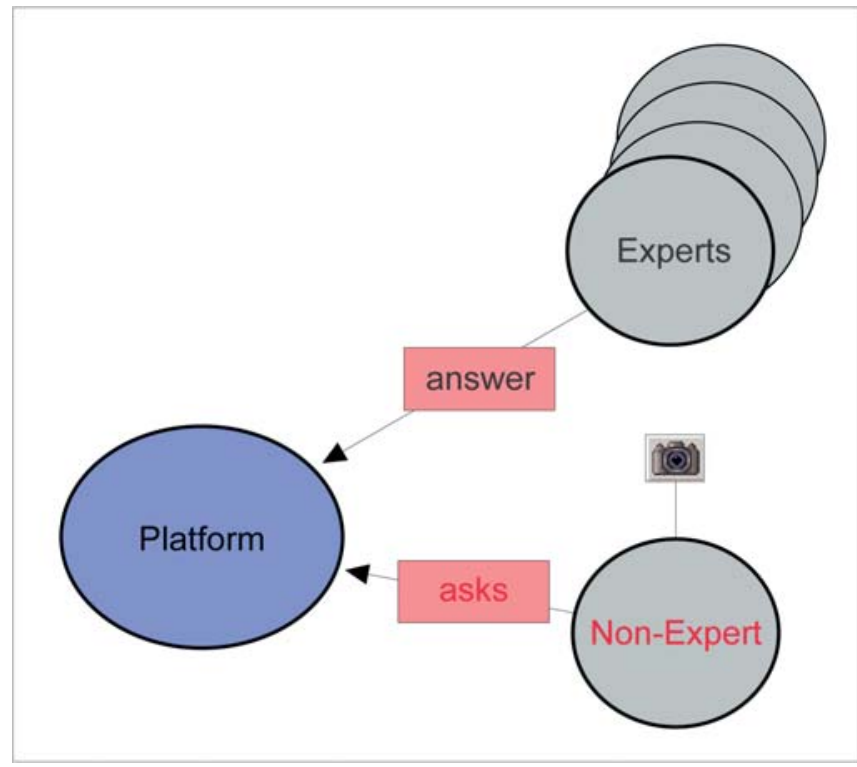

Fig. 1 Telepathology is a structured dialogue. Technically, the non-expert deposits his questions and images on a server (platform). The experts can see what is stored in the database on the server and can add their comments. The non-expert can re-contact the server and see the answers given

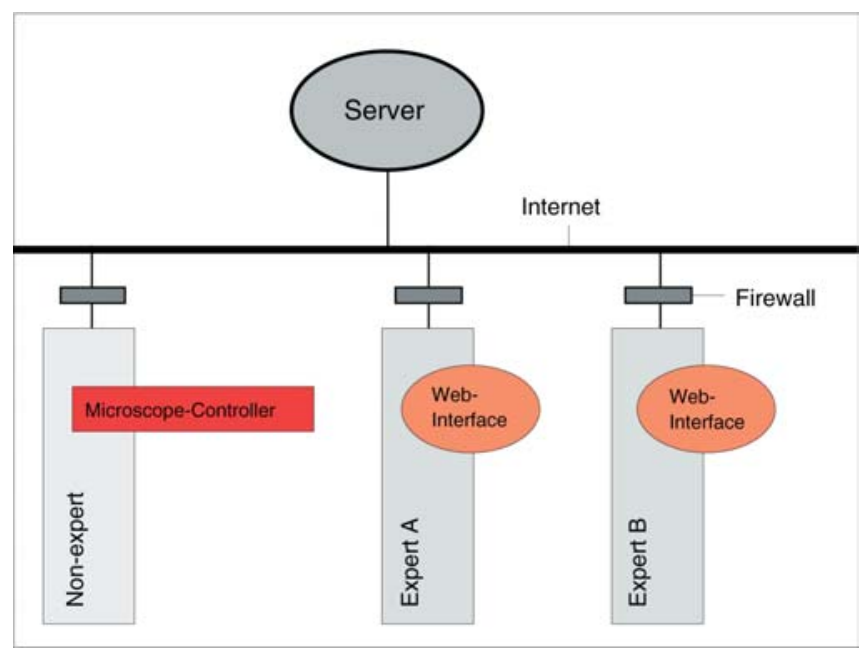

Fig. 2 The location of the server determines the user-friendliness of access to the system. The iPath-Server is in the Internet without access restriction from any firewall. This position guarantees easy access for every authorised user at any time or location

larisation of single hardware elements and software elements for the different tasks.

The most important module for implementing an efficient telepathology is the Internet. Since Internet browsers and e-mail clients usually exist on all computers, an expert can easily join and participate in a discussion group (even for remote microscopy). The nonexpert will only need a digital camera or corresponding appliance connected to his or her computer. 
The first application of Internet browser technology for telemicroscopy or dynamic telepathology $[1,8,16,24]$ was demonstrated by the TeleMic project at the Charitée University of Berlin [19, 27]. However, a fundamental problem in using the Internet is firewalls, which are installed in the different institutions, prohibiting a spontaneous and direct connection between computers located within the institution and computers outside of the institution. The most convenient solution for this problem is the use of a server directly located in the Internet and directing all traffic "between two or more computers (clients)" through that server [3,21] (Fig. 2).

\section{The "conceptional" summary of over $\mathbf{1 0}$ years experience in telepathology (telemedicine)}

The newly developed telemedical system iPath is the result of broad practical experiences and theoretical analyses in many fields of telemedicine. The first experiences were in remote microscopy [18]. iPath implements characteristics of both a collaboration tool and a content management system. The content that is managed is the questions of the non-experts (texts, images and/or other objects) and the answers of the experts.

The centre of each telepathology system: the database

The relational database allows the collection of all data transferred between the partners. Around this core is the application governing content, an application that currently has a web- and a (standardised) e-mail-interface implemented. The content managed is divided into discussion or expert groups (see above). The importance of a clear database concept as the basis of a telepathology system for routine use is, however, referred to in only a very few publications $[18,21]$.

Within the groups in the database are the cases (records) (Fig. 3). Finally, each case contains the actual data produced: the questions, the "objects" (images, schemas, forms, other relevant documents or textual information), and the comments (diagnoses, remarks, additional questions). This central storage of data with search facilities serves as an archive of all past collaborations and consultations, if necessary. If the remote microscopy module is used, the session is linked to an open case defined beforehand within a pre-designed group, and the expert can choose to add a selection of the pictures transmitted via the special module from the microscope to the video eye on the server. For real-time conferencing, an online chat function can be used.

Access to the "world of telepathology"

A group on a server is administered automatically. A potential group member must first register an account for

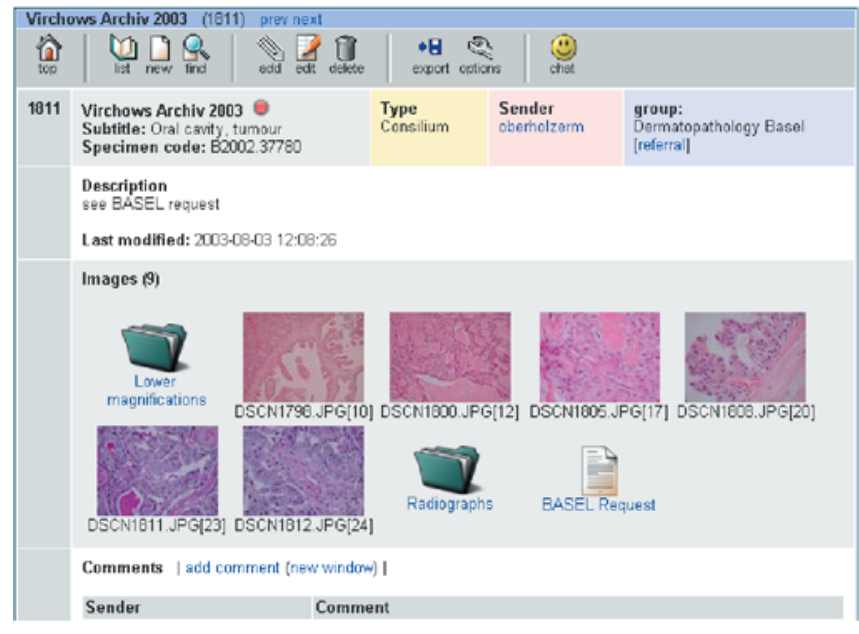

Fig. 3 The database consists of groups and cases (records). A case is stored within a group. Each case is subdivided into four segments: identification, description (free text), images (gallery of documents) and comments

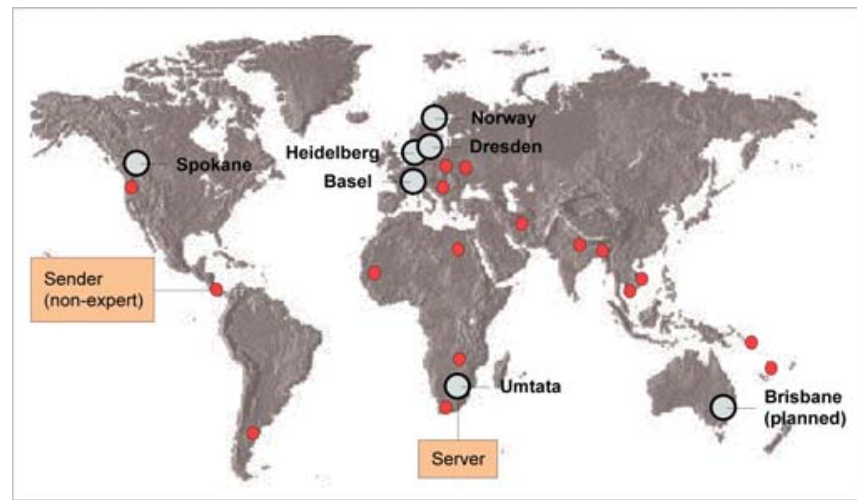

Fig. 4 Use of iPath around the world. iPath runs on six servers around the world. The server in Norway serves a health network in Western Africa. The server in Dresden functions as a centre of a field study about breast carcinoma organised by the German State of Sachsen

the server and then contact the relevant group administrator for a group membership.

An iPath-Server can be installed using freely available open source software tools, such as Apache for the webinterface, and can run on the free operating system, Linux. This approach has been taken by several institutions that want to keep autonomy over their data [West African Doctors Network, Inland Northwest Health Service for the Spokane-district (Washington State, USA), breast carcinoma field studies in Dresden, Germany] (Fig. 4). Among the security measures implemented in our system are automatic interruption of open connections without activity after a predefined time and the possibility to encrypt all traffic to the server. 


\section{Main telemedical tasks}

General observations

In September 2001, iPath was released and opened on the main server in Basel, with the intention to empirically observe in which direction the applications of such an open telepathology system would develop. The results are represented as follows.

In contrast to other Internet-based telepathology systems $[9,26]$, iPath does not impose a predefined workflow or mode of collaboration. It is, rather, a platform on which telemedical consultations and other applications can individually be arranged. The telemedical server in Basel is now used by over 800 users organised in more than 70 discussion or expert groups.

\section{Consultations in pathology}

Consultation is one of the main uses for a telemedical application. A recent evaluation showed that $43.8 \%$ of all cases on the telemedicine server in Basel were consultations in pathology. Consultations in telehistology and telecytology are desired by: (1) institutions without a pathologist, e.g. the Referral Hospital of Honiara, Solomon Islands (South Pacific); (2) general pathologists who work alone as pathologists in a hospital, e.g. the Department of Pathology of the Dhaka Medical College, Bangladesh or (3) pathologists in a speciality, e.g. at the University Hospital of Teheran, Iran, or the Working Group of Bone Tumours of Austria, Germany and Switzerland.

A very interesting result deduced of our practical experiences in the last 2 years is the Virtual Institute of Pathology (VIRIN). VIRIN represents an organised coworking of many experts distributed over the whole world. These experts work with different institutions. The members of these institutions are non-experts. The experts are members of their own expert group, as well as of the discussion (sender) groups of the non-experts. The non-experts submit their cases in their discussion group. In the VIRIN, one expert of the expert group is always on duty. He monitors the discussion groups (e.g. "South Pacific" for Honiara). He is responsible for a response to cases submitted by Honiara in the "South Pacific" group within 48-60 h. This expert can decide to present a newly submitted case to the other experts in the VIRIN. In the VIRIN, the case can be freely discussed without the nonexperts seeing the different comments of the experts on his case. A summary of the discussion of the experts is then added to the original case (in the discussion group) by the expert on duty.

The results of the collaboration with Honiara in the time between 26 September 2001 and 2 May 2003 were analyzed under different aspects. One aspect was that, in the observed period, the percentage of benign diagnoses increased, approaching, in the phase where the VIRIN for Honiara was fully established, an amount as one would

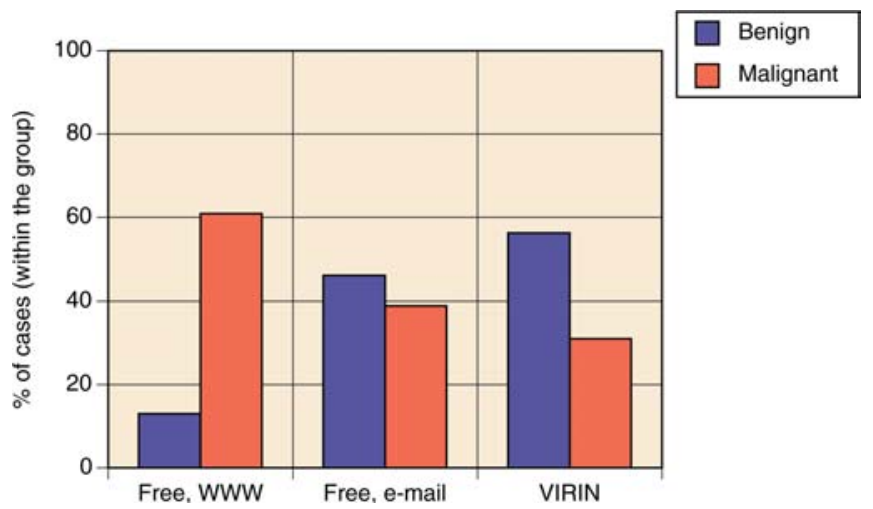

Fig. 5 The percentage of benign and malignant diagnoses. The collective "Free, WWW" consists of 23 cases (Period: 26 September 2001-19 March 2002), the collective "Free, e-mail" of 54 (Period: 20 March 2002-30 Setpember 2002), and the collective "VIRIN" of 71 cases (Period: 1 November 2002-2 May 2003). The submission per e-mail was carried out by a standardised specially developed e-mail procedure. Chi2 value: $10.062, v=2, P<0.01$

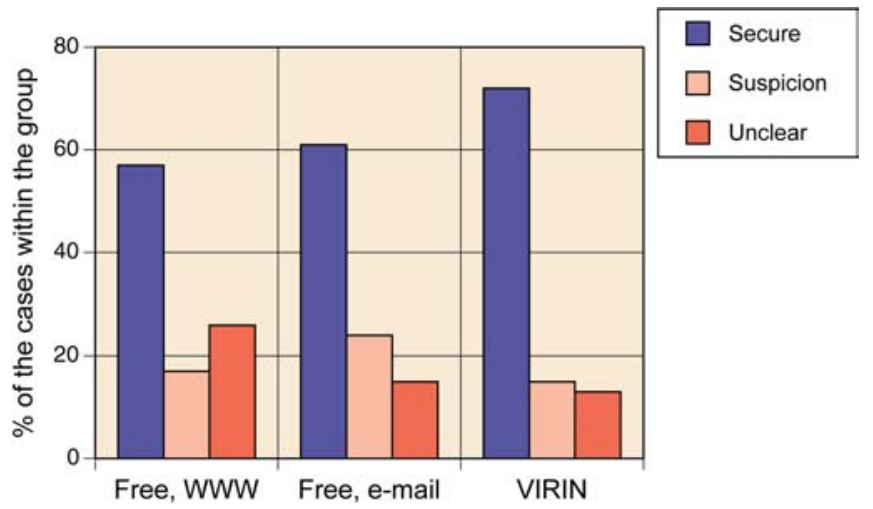

Fig. 6 The percentage of secure, suspicious and unclear diagnoses. Chi 2 value: $4.142, v=4, P<0.20$ )

expect at a typical Western pathology institution (Fig. 5). This indicates that, after an initial phase of deciding only to discuss cases that mostly were assumed to be malign, Honiara is now comfortable with having an assessment of all the cases a Western hospital would usually submit. The introduction of the VIRIN also improved the percentage of secure diagnoses as opposed to those where doubts remained (Fig. 6).

\section{Interdisciplinary conferences}

The tumour board of the Regional Hospital of Lörrach (Germany, near Basel) has been running systematically on iPath for 1.5 years. When preparing the tumour board, the key information from contributing institutions (e.g. pathology or radiology) is added to the cases that are previewed by the specialists before the conference. 
Health networks

It could be observed that our telemedical system can simultaneously be used efficiently for both diagnosis and e-learning. This quality led to the idea to use iPath as a nucleus of health networks in the Ukraine (in collaboration with the Swiss Tropical Institute) and in the Eastern Cape province (South-Africa).

\section{Field studies}

A surprising result was the application of the actual system for field studies without special adaptations. In such studies, the user needs are the inverse of the original needs in telepathology, where knowledge is provided by a centre to a peripheral institution. The key element allowing this new application is forms. Forms correspond to a media record with empty text fields and can be created individually. The forms are stored as an integral part of the group's record structure. The content of all the forms can be exported as a two-dimensional textfile for further evaluation locally.

\section{Implications after more than $\mathbf{1 0}$ years experience from a single institution}

In the last years, we and others intensively experimented with an open telemedical system. The most important aspects which crystallised in using telepathology were: (1) working in virtual groups of specialists (experts), (2) a categorisation of telepathology in four main fields, (3) a definition of new (minimal) standards and (4) some hints as to change the general conception of telepathology (paradigm change).

\section{The virtual group model (VIRIN)}

The virtual group model was postulated by Weinstein et al. [24]. An open telepathology system allows a successful implementation of this model, of which the objective has become a reality with the Honiara project. Our VIRIN shows the necessity to clearly organise the diagnostic telepathological work and to exactly distinguish the role of all partners. Additionally, virtual working groups can give rise to a new vision of the job of a pathologist: telepathology offers retired professionals the possibility of remaining in their professional activity and allows society to continue to profit from the great experience of retired pathologists.

\section{Categorisations in telepathology}

Commenting on subdivisions discussed in previous publications on telepathology [10, 15, 16, 17, 18, 23], we see that the main focus has been on technical aspects of the method. In the early days of telepathology, the main differentiation was made between dynamic-robotic [1, 12 , 20] versus static telepathology [5, 6, 20] models, which were mainly applied to point-to-point diagnostic telepathology links. With the advancement of Internetbased telepathology, the workflow process was brought much more into the centre of discussion. Weinstein et al. [24] suggest three categories of workflow organisation, namely the case triage model, subspeciality model and the virtual group practice model.

On the background of our observations and experiences, we propose to discriminate the following four telepathological categories: consultations, tumour boards, field studies/research and educational applications (Table 1). The three workflow models proposed by Weinstein et al. [24] can all be implemented through organising telepathology by discussion groups, optionally linked to an expert group.

\section{Change of paradigm}

Modern communication technologies, first and foremost the Internet, have brought telepathology and telemedicine a decisive step forward: a step that anticipates a change of paradigm, a change away from our current constellation of convictions, views and methods [13].

Technically, the field of telemedicine was dominated, until recently, by individual solutions, which could not communicate with each other. With the idea "iPath", we pursued the goal to give non-experts the means of remote dialogue with experts, using as much as possible of the non-experts existing infrastructure. Much of the task was: (1) to intelligently combine the computer resources with a database, (2) to achieve a high degree of organisation in their practical use and (3) to design the whole system as a modular content management system.

We can expect that convictions and methods in pathology will change in relation to the simplicity and spontaneity of telepathology in the future. Because telepathology-especially in rural areas $[7,28,29,30]-$ is increasingly being used by institutions without resident pathologists, the non-pathologist must become more familiar with the evaluation of macroscopy, the sampling of tissue specimens and the selection of microscopic fields.

\section{Standardisation}

Any broadly implemented method calls for some basic regulation $[2,14]$. The probability of a minimal standardisation grows at the rate with which we succeed in clearly defining the technical elements of the method and precisely structuring the processes. The question of standardisation can be reduced to deciding on a serverclient technology, the minimal requirements and structure for the database and the key features of the module's data input and output. 
Table 1 The four new main application categories in telepathology. The spontaneous use of iPath has demonstrated that the different applications can be allocated to four main categories: (1) consultations, (2) tumour boards, casuistics/clinical pathological conferences (CPCs), (3) lectures and (4) field studies

\begin{tabular}{|c|c|c|c|c|}
\hline \multirow[t]{2}{*}{ Authors } & \multirow{2}{*}{$\begin{array}{l}\text { Applications } \\
\text { (Content) }\end{array}$} & \multirow{2}{*}{$\begin{array}{l}\text { Model } \\
\text { (Organisation) }\end{array}$} & \multicolumn{2}{|c|}{$\begin{array}{l}\text { Activity of the experts in relation } \\
\text { to the non-experts }\end{array}$} \\
\hline & & & At the same time ${ }^{1}$ & Time-shifted \\
\hline \multirow[t]{7}{*}{ [24] } & $\begin{array}{l}\text { Intraoperative frozen } \\
\text { section diagnosis }\end{array}$ & & + & \\
\hline & $\begin{array}{l}\text { Surgical pathology } \\
\text { consultation }\end{array}$ & & & + \\
\hline & $\begin{array}{l}\text { Expert-to-expert } \\
\text { consultations }\end{array}$ & & & + \\
\hline & Distance education & & & + \\
\hline & & Subspeciality & & \\
\hline & & Case triage & & \\
\hline & & Virtual group & & \\
\hline \multirow{11}{*}{$\begin{array}{l}\text { Present } \\
\text { paper }\end{array}$} & Consultations & Discussion group & + & \\
\hline & Active & & & \\
\hline & Frozen sections & & & \\
\hline & $\begin{array}{l}\text { Conventional diagnosis } \\
\text { Others }\end{array}$ & & & \\
\hline & Passive & Virtual institute & & + \\
\hline & Tumour boards & Discussion group & & + \\
\hline & Field studies & Discussion groups & & + \\
\hline & Distance education & Discussion groups & & + \\
\hline & Casuistics & & & \\
\hline & CPCs & & & \\
\hline & e-learning & & & \\
\hline
\end{tabular}

${ }^{1}$ iPath offers a chat function, which can be spontaneously used
Telepathology: a modern win-win situation for everyone?

Empirically, we could clearly show that telemedicine as an "open distributed system" based on client-server technology could perform according to the needs of users in a society saturated with information technology, as well as in an environment with minimal communication infrastructure. Open telemedicine allows these environments to be tied together and, thus, generates an added value for all partners.

\section{References}

1. Baak JP, van Diest PJ, Meijer GA (2000) Experience with a dynamic inexpensive video-conferencing system for frozen section telepathology. Anal Cell Pathol 21:169-175

2. Belnap CP, Freeman JH, Hudson DA, Person DA (2002) A versatile and economical method of image capture for telepathology. J Telemed Telecare 8:117-120

3. Brauchli K, Christen H, Haroske G, Meyer W, Kunze KD, Oberholzer M (2002) Telemicroscopy by the Internet revisited. J Pathol 196:238-243

4. Brauchli K, Helfrich M, Christen H, Jundt G, Haroske G, Mihatsch M, Oberli H, Oberholzer M (2002) [The future of telepathology. An Internet "distributed system" with "open standards"]. Pathologe 23:198-206

5. Cross SS, Dennis T, Start RD (2002) Telepathology: current status and future prospects in diagnostic histopathology. Histopathology 41:91-109

6. Della Mea V, Cataldi P, Boi S, Finato N, Della Palma P, Beltrami CA (1998) Image selection in static telepathology through the Internet. J Telemed Telecare 4[Suppl 1]:S20-S22

7. Della Mea V, Cortolezzis D, Beltrami CA (2000) The economics of telepathology - a case study. J Telemed Telecare 6[Suppl 1]:S168-S169

8. Demichelis F, Barbareschi M, Boi S, Clemente C, Dalla Palma P, Eccher C, Forti S (2001) Robotic telepathology for intraop- erative remote diagnosis using a still-imaging-based system. Am J Clin Pathol 116:744-752

9. Dietel M, Nguyen-Dobinsky TN, Hufnagl P (2000) The UICC Telepathology Consultation Center. International Union Against Cancer. A global approach to improving consultation for pathologists in cancer diagnosis. Cancer 89:187-191

10. Dunn BE, Almagro UA, Choi H, Sheth NK, Arnold JS, Recla DL, Krupinski EA, Graham AR, Weinstein RS (1997) Dynamic-robotic telepathology: Department of Veterans Affairs feasibility study. Hum Pathol 28:8-12

11. Furness PN, Bamford WM (2001) Telepathology. Review. Curr Diagn Pathol 7:281-291

12. Gombas P, Skepper JN, Hegyi L (2002) The image pyramid system-an unbiased, inexpensive and broadly accessible method of telepathology. Pathol Oncol Res 8:68-73

13. Küng H (2002) Erkämpfte Freiheit. Erinnerungen. Pieper Verlag $\mathrm{GmbH}$, München

14. Loane M, Wootton R (2002) A review of guidelines and standards for telemedicine. J Telemed Telecare 8:63-71

15. Nordrum I (1998) Real-time diagnoses in telepathology. Adv Clin Pathol 2:127-131

16. Nordrum I, Eide TJ (1995) Remote frozen section service in Norway. Arch Anat Cytol Pathol 43:253-256

17. Oberholzer M, Fischer HR, Christen H, Gerber S, Bruhlmann M, Mihatsch M, Famos M, Winkler C, Fehr P, Bechthold L (1993) Telepathology with an integrated services digital network-a new tool for image transfer in surgical pathology: a preliminary report. Hum Pathol 24:1078-1085

18. Oberholzer M, Fischer HR, Christen H, Gerber S, Bruhlmann M, Mihatsch MJ, Gahm T, Famos M, Winkler C, Fehr P (1995) Telepathology: frozen section diagnosis at a distance. Virchows Arch 426:3-9

19. Person DA (2000) Pacific Island Health Care Project: early experiences with a Web-based consultation and referral network. Pac Health Dialog 7:29-35

20. Remmelinck M, Lopes MB, Nagy N, Rorive S, Rombaut K, Decaestecker C, Kiss R, Salmon I (2000) How could static telepathology improve diagnosis in neuropathology? Anal Cell Pathol 21:177-182 
21. Rogers N, Furness P, Rashbass J (2001) Development of a lowcost telepathology network in the UK National Health Service. J Telemed Telecare 7:121-123

22. Stredney D, Crawfis R, Wiet GJ, Sessanna D, Shareef N, Bryan J (1999) Interactive volume visualizations for synchronous and asynchronous remote collaboration. Stud Health Technol Inform 62:344-350

23. Weinberg DS, Allaert FA, Dusserre P, Drouot F, Retailliau B, Welch WR, Longtine J, Brodsky G, Folkerth R, Doolittle M (1996) Telepathology diagnosis by means of digital still images: an international validation study. Hum Pathol 27: 111-118

24. Weinstein RS, Descour MR, Liang C, Bhattacharyya AK, Graham AR, Davis JR, Scott KM, Richter L, Krupinski EA, Szymus J, Kayser K, Dunn BE (2001) Telepathology overview: from concept to implementation. Hum Pathol 32:1283-1299
25. Wells CA, Sowter C (2000) Telepathology: a diagnostic tool for the millennium? J Pathol 191:1-7

26. Williams BH (1998) The AFIP center for telemedicine application-pathology for the twenty-first century. Telemed Virtual Real 3:64-65

27. Wolf G, Petersen I, Dietel M (1998) Microscope remote control with an Internet browser. Anal Quant Cytol Histol 20:127-132

28. Wootton $\mathrm{R}$ (1997) The possible use of telemedicine in developing countries. J Telemed Telecare 3:23-26

29. Wright D (1997) Telemedicine and developing countries: a report of Study Group 2 of the ITU Development Sector. $\mathrm{J}$ Telemedicine Telecare 4:1-85

30. Zhao Y, Nakajima I, Juzoji H (2002) On-site investigation of the early phase of Bhutan Health Telematics Project. J Med Syst 26:67-77 